\title{
Single and double addition of oxygen atoms to propyne on surfaces at low temperatures
}

\author{
Helen J. Kimber, Courtney P. Ennis† and Stephen D. Price*
}

Received 11th December 2013, Accepted 14th January 2014

DOI: $10.1039 / c 3 f d 00130 j$

\begin{abstract}
Experiments designed to simulate the low temperature surface chemistry occurring in interstellar clouds provide clear evidence of a reaction between oxygen atoms and propyne ice. The reactants are dosed onto a surface held at a fixed temperature between 14 and $100 \mathrm{~K}$. After the dosing period, temperature programmed desorption (TPD), coupled with time-of-flight mass spectrometry, are used to identify two reaction products with molecular formulae $\mathrm{C}_{3} \mathrm{H}_{4} \mathrm{O}$ and $\mathrm{C}_{3} \mathrm{H}_{4} \mathrm{O}_{2}$. These products result from the addition of a single oxygen atom, or two oxygen atoms, to a propyne reactant. A simple model has been used to extract kinetic data from the measured yield of the singleaddition $\left(\mathrm{C}_{3} \mathrm{H}_{4} \mathrm{O}\right)$ product at surface temperatures from 30-100 K. This modelling reveals that the barrier of the solid-state reaction between propyne and a single oxygen atom $(160 \pm 10 \mathrm{~K})$ is an order of magnitude less than that reported for the gas-phase reaction. In addition, estimates for the desorption energy of propyne and reaction rate coefficient, as a function of temperature, are determined for the single addition process from the modelling. The yield of the single addition product falls as the surface temperature decreases from $50 \mathrm{~K}$ to $30 \mathrm{~K}$, but rises again as the surface temperature falls below $30 \mathrm{~K}$. This increase in the rate of reaction at low surface temperatures is indicative of an alternative, perhaps barrierless, pathway to the single addition product which is only important at low surface temperatures. The kinetic model has been further developed to characterize the double addition reaction, which appears to involve the addition of a second oxygen atom to $\mathrm{C}_{3} \mathrm{H}_{4} \mathrm{O}$. This modelling indicates that this second addition is a barrierless process. The kinetic parameters we extract from our experiments indicate that the reaction between atomic oxygen and propyne could occur under on interstellar dust grains on an astrophysical time scale.
\end{abstract}

\section{Introduction}

The elemental composition of the known universe comprises almost exclusively light atoms ( 99.9\% hydrogen and helium). However, to date, over 160 different 
molecules have been detected in the interstellar medium. ${ }^{1}$ The vast majority of these interstellar molecules contain hydrogen. The distribution of these molecules is far from uniform across the interstellar medium (ISM), where dense interstellar clouds are observed to harbour relatively large densities and varieties of complex molecules that have been proposed as precursors to biologically relevant species., ${ }^{2,3}$ In these interstellar clouds, molecular lifetimes are extended with respect to other more diffuse parts of the ISM. Specifically, the relative opacity of these dense interstellar clouds shields the cloud's centre from high energy photons, allowing relatively fragile molecules to survive for extended periods.

In dense interstellar clouds, molecules are detected in abundances that cannot be completely accounted for by known gas-phase kinetics. ${ }^{4}$ Hence, it is now widely accepted that there is a contribution to these molecular abundances from reactions on and within the molecular ices on the surfaces of interstellar dust. This interstellar dust comprises predominantly silicate or carbonaceous particles, with a typical diameter of $100 \mathrm{~nm},{ }^{5}$ making up typically $1 \%$ of the mass of an interstellar cloud. ${ }^{6}$

In general, bimolecular reactions on surfaces are thought to follow one of two prototypical reaction pathways. The first is the Langmuir-Hinshelwood (LH) mechanism where both reactive species are initially adsorbed and thermalised on the surface. ${ }^{7}$ Diffusive processes then allow the reactants to encounter one another and react. The second mechanism, the Eley-Rideal (ER) pathway, involves a thermalised surface molecule undergoing direct reaction with an incident, and potentially energetic, gas-phase species. ${ }^{7}$ To correctly model the contribution of these heterogeneous reactions to the abundances of interstellar molecules, kinetic data is required. The measurement of such kinetic data is the objective of our experimental efforts. ${ }^{\mathbf{8} 9}$

One relatively abundant interstellar molecule, that is considered to play an important role in astrochemical processes, is the small unsaturated hydrocarbon, propyne $\left(\mathrm{CH}_{3} \mathrm{CCH}\right) .{ }^{10,11}$ Interstellar propyne was first identified in the Milky Way in the giant molecular cloud Sagittarius B2. ${ }^{11,12}$ Propyne has also been observed in cold cloud cores and lukewarm corinos in the Milky Way. ${ }^{13}$ Propyne has also been detected across the disc of the distant Messier 82 (M82) galaxy. ${ }^{14}$ In M82, the propyne-to-methanol ratio $\left(\left[\mathrm{CH}_{3} \mathrm{CCH}\right] /\left[\mathrm{CH}_{3} \mathrm{OH}\right]>8\right)$ is higher than for comparable starburst galaxies such as NGC $253\left(\left[\mathrm{CH}_{3} \mathrm{CCH}\right] /\left[\mathrm{CH}_{3} \mathrm{OH}\right] \approx 1\right) .{ }^{15}$ The propyne component of a prototypical starburst galaxy such M82 and NGC 253 resides primarily in interstellar clouds, where thermal dust grain chemistry is potentially important. Here we distinguish thermal surface chemistry, where reactions occur on the surface without the input of additional energy, with activated chemistry, where reactions are initiated and molecules subsequently processed, by external agents such as cosmic rays. ${ }^{16}$

To our knowledge, despite propyne being repeatedly detected in the ISM, the reactivity of propyne on cold surfaces, such as those of dust grains, has not yet been investigated. In contrast, the chemistry of methanol on interstellar surfaces has been extensively studied. ${ }^{17-20}$ Given their comparable abundances, the surface reactivity of propyne appears to be overdue an investigation. Such an investigation is the target of this study.

In addition to its detection in interstellar clouds, propyne has also been observed in planetary environments in our Solar System. Specifically, propyne has 
been detected in stratospheres of Jupiter and Saturn, displaying column densities of $1.5 \pm 4 \times 10^{14}$ molecules $\mathrm{cm}^{-2}$ and $2 \times 10^{15}$ molecules $\mathrm{cm}^{-2}$ respectively. ${ }^{21,22}$ Interstellar ice composition is considered to be important when discussing the abundance of propyne in the Saturnian and Jovian atmospheres. Here, volatile molecules, such as propyne, are thought to originate from molecules trapped as clathrates in the water ice that aggregated during the formation of the planetisimals that preceded these planets. ${ }^{23-28}$ Propyne has also recently been identified in the atmosphere of Uranus ${ }^{29}$ and the atmosphere of Saturn's moon Titan, where it appears enriched at northern latitudes. ${ }^{30,31}$ In all cases propyne is identified by its infrared absorption band $\left(v_{9}\right)$ centred at $15.8 \mu \mathrm{m}$.

Oxygen is the third most abundant element in the ISM, after hydrogen and helium. ${ }^{32}$ There has been considerable recent interest in accounting for the relatively low abundance (the so-called depletion) of oxygen in the gas-phase in the interstellar medium. ${ }^{3-35}$ It has been proposed that interstellar grains could act as a sink for oxygen atoms, but it appears that the necessary depletion cannot be generated simply by the incorporation of oxygen atoms into the structures of the dust grains themselves. ${ }^{34}$ One possible additional sink for oxygen atoms is perhaps their reaction with the organic component of the molecular ice mantles that coat the dust grains in some interstellar clouds. The reaction we investigate in this paper, the addition of oxygen atoms to propyne on a cold surface, could be considered representative of oxygen atom depletion by reaction with organic ices.

Oxygen atom diffusion has recently been studied on an amorphous water ice surface. ${ }^{36}$ In this work Minissale et $a l^{36}$ report that oxygen atom diffusion exhibits a quantum-classical transition at a surface temperature of $20 \mathrm{~K}$. That is, above $20 \mathrm{~K}$ a classical motion involving barrier 'hopping' between adsorption sites is the dominant $\mathrm{O}$ atom migration mechanism, but at surface temperatures below $20 \mathrm{~K}$ quantum tunnelling of the $\mathrm{O}$ atoms becomes dominant.

The gas-phase reaction of propyne with $\mathrm{O}$ atoms has been studied both experimentally and computationally. ${ }^{37-44}$ In the gas phase the reaction produces $\mathrm{CO}$ and the $\mathrm{CH}_{3} \mathrm{CH}$ diradical which form from a vibrationally excited methyl ketene..$^{45}$ At high pressures, or in a condensed medium where intermediates can be collisionally stabilized, the adduct that results from $\mathrm{O}$ atom addition is expected to simply relax to a more stable isomer such as methyl ketene or propenal..$^{38,46}$

In this paper we present a study of the reaction of propyne with atomic oxygen on a cold surface. These experiments generate kinetic data for this reaction under astrophysically relevant conditions. These studies build on our previous investigations of the reaction of oxygen atoms with alkenes ${ }^{9}$ and sulphur containing molecules. ${ }^{8}$

\section{Experimental procedure}

The experimental apparatus employed in this study, which has been described in detail before, ${ }^{8,9}$ is designed to probe the reactions of molecules with atomic species on molecular ices deposited on a graphite surface held at a specific temperature. The surface temperatures investigated are pertinent to heterogeneous reactivity on dust surfaces in the ISM. The products of any surface reactions are detected by a temperature-programmed desorption (TPD) methodology. In the TPD experiment, either an electron beam or a laser is used to ionize the molecules 


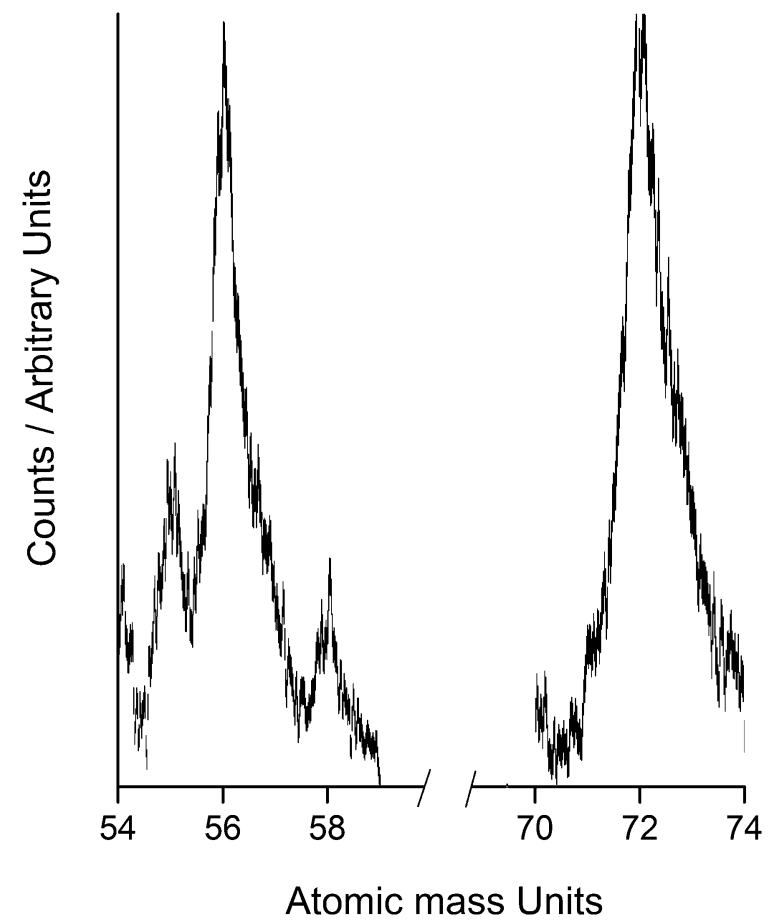

Fig. 1 Sections of representative mass spectra recorded during the TPD phase showing peaks for $\mathrm{m} / \mathrm{z}=56$ and $\mathrm{m} / \mathrm{z}=72$, corresponding to the single and double addition products. See text for details.

formed on the surface when they are desorbed as the surface is heated. The ions resulting from this ionization of the desorbed molecules are detected and identified by a time-of-flight mass spectrometer (TOFMS). ${ }^{47}$

The experimental apparatus consists of two vacuum chambers. The 'source' chamber has a base pressure of approximately $10^{-7}$ Torr. This chamber houses a microwave discharge cell used to generate $\mathrm{O}$ atoms from $\mathrm{O}_{2}$. Previous experiments have determined an $\mathrm{O}_{2}$ dissociation efficiency of approximately $20 \%$ from this source, ${ }^{9}$ so $\mathrm{O}_{2}$ is still the majority species in our "O atom beam".

In investigating the low-temperature reactions induced on surfaces by our $\mathrm{O}$ atom source one must be aware, as discussed before, ${ }^{9}$ that ozone can be formed by reactions of $\mathrm{O}$ atoms with $\mathrm{O}_{2}$ on the surface. However, the oxygen atom addition reactions we observe proceed efficiently at surface temperatures well above $30 \mathrm{~K}$, where ozone is not present on the surface. ${ }^{9}$ This observation indicates that the oxygenated products we observe are not the result of the reaction between propyne and ozone. Supporting this analysis is the observation that ozone only reacts with unsaturated hydrocarbons at low temperatures following irradiation. ${ }^{48}$ We also note that no reaction is observed if no microwaves are applied to the $\mathrm{O}$ atoms source and simply $\mathrm{O}_{2}$ is dosed onto the surface.

Gas from the $\mathrm{O}$ atom source, undergoes significant differential pumping in the source chamber before being piped into the 'target' chamber. A second deposition line attached to the source chamber allows the dosing of stable molecules, in this case propyne, onto the cold surface in addition to the oxygen atoms. Again, 


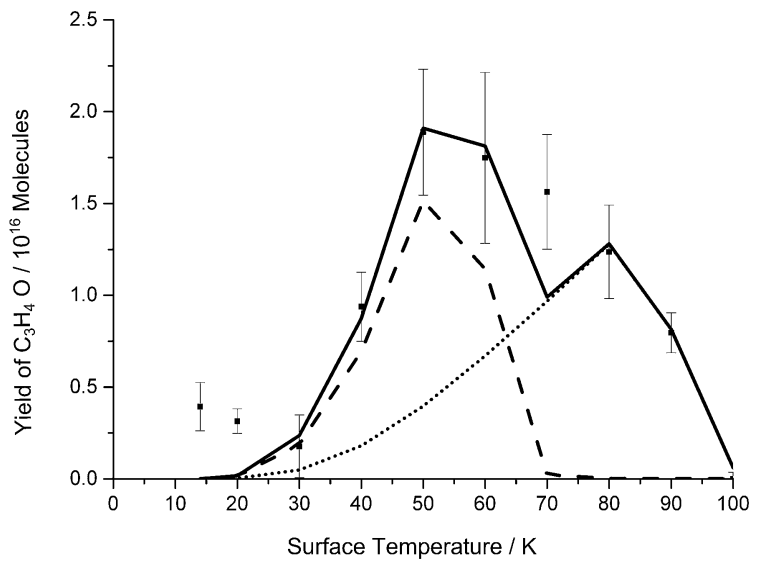

Fig. 2 Summed yield of the single and double addition product, formed following the codeposition of propyne $\left(\mathrm{C}_{3} \mathrm{H}_{4}\right)$ and $\mathrm{O}$ atoms, as a function of surface temperature. Squares: experimental data; solid line: model; dash LH mechanism; dot ER mechanism. The error bars associated with the experimental results represent two standard deviations from four repeats at each surface temperature. The kinetic model used to derive the fit shown employs the paramenters listed in Table 1 and an $\bigcirc$ atom desorption energy of $15.3 \mathrm{~kJ}$ $\mathrm{mol}^{-1}$.

the propyne dosing line undergoes significant differential pumping in the source chamber before being directed into the target chamber. This differential pumping arrangement serves to allow sufficient pressure in the microwave source for stable operation, whilst dosing the target surface at an acceptably low rate. The UHV target chamber has a base pressure of approximately $10^{-10}$ Torr when the dosing gases are switched off, but has a pressure of approximately $10^{-8}$ Torr during the dosing process.

The PTFE tubes which direct the reactants from the source chamber into the target chamber terminate close above a highly oriented pyrolytic graphite (HOPG) substrate. This substrate can be cooled to close to $10 \mathrm{~K}$ and heated to above $500 \mathrm{~K}$. Propyne and $\mathrm{O}_{2} / \mathrm{O}^{\prime}$ are co-deposited on the HOPG substrate at a fixed surface temperature (in the range from $14 \mathrm{~K}$ to $100 \mathrm{~K}$ ). The fluxes of the propyne and $\mathrm{O}$ atoms are $1.0 \times 10^{15} \mathrm{~cm}^{-2} \mathrm{~s}^{-1}$ and $1.3 \times 10^{14} \mathrm{~cm}^{-2} \mathrm{~s}^{-1}$ respectively. ${ }^{9}$ After one hour, the delivery lines are evacuated and the substrate is allowed to cool from the fixed deposition temperature to $14.0 \pm 0.5 \mathrm{~K}$. A current is then passed through a tantalum strip heater to slowly raise the surface temperature to $\sim 200 \mathrm{~K}$. As the substrate temperature increases, the molecules on the surface desorb at their specific sublimation temperatures and enter the source region of the TOFMS which is located in front of the sample. It should be noted that the dosing fluxes employed in our experiments rapidly result in a multilayer propyne $/ \mathrm{O}_{2} / \mathrm{O}$ ice. Thus, formally the reactivity we observe should be considered representative of this surface. However, since the propyne molecules are physisorbed at the surface, we can assume that their electronic structure is largely unperturbed upon adsorption, and thus the reactivity we observe should be broadly representative of physisorbed propyne, irrespective of the precise nature of the surface on which the molecule is adsorbed.

Molecules desorbed from the surface are ionized in the source region of the TOFMS by a pulsed beam of $200 \mathrm{eV}$ electrons running with a duty cycle of $32 \mu \mathrm{s}$. 


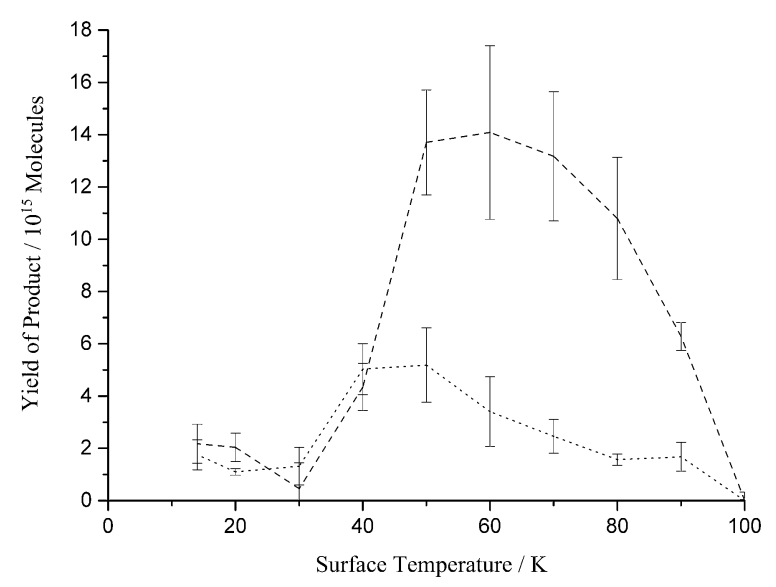

Fig. 3 Experimental yield of the single (dashed line) and double (dotted line) addition products following the co-deposition of propyne $\left(\mathrm{C}_{3} \mathrm{H}_{4}\right)$ and $\mathrm{O}$ atoms. The error bars associated with the experimental results represent two standard deviations from four experiments at each surface temperature. The lines linking the points serve only to guide the eye.

The electron pulses have a duration of approximately $1 \mu \mathrm{s}$. After each pulse of electrons crosses the source, a voltage is applied to the repeller plate of the TOFMS to accelerate any ions formed in the spectrometer's source region towards the detector for analysis. The detector is a pair of multichannel plates (MCPs), the output from which is amplified and discriminated before being passed to a timeto-digital convertor (TDC). The TDC is triggered by the same pulse generator that controls the electron gun and the repeller plate voltage. Times from the TDC are recorded as a histogram of time-of-flight versus counts, i.e. a mass spectrum. During the TPD phase, as the temperature of the surface is slowly increased, a new mass spectrum is generated every second. At the end of each TPD experiment, these mass spectra are combined to generate a two-dimensional data set of ion intensity at each mass-to-charge $(\mathrm{m} / \mathrm{z})$ ratio against the substrate temperature during the heating process. This dataset can then be used to generate, for example, a mass spectrum for a particular temperature range or to see how the intensity of a particular ion (at a given $\mathrm{m} / \mathrm{z}$ ) varies as the surface temperature increases. In this work we recorded these TPD datasets after dosing propyne and $\mathrm{O}$ atoms onto the surface at a range of surface temperatures between $14 \mathrm{~K}$ and $100 \mathrm{~K}$, performing four separate dosing/TPD measurements at each surface temperature investigated.

\section{Results}

Whenever propyne and $\mathrm{O}$ atoms are allowed to react at surface temperatures below $100 \mathrm{~K}$, we observe signals at mass-to-charge ratios $(\mathrm{m} / \mathrm{z})$ of 56 and 72 in the TOF mass spectra recorded during the TPD phase (Figure 1). These two signals possess different TPD profiles, with the signal at $m / z=56$ appearing at lower desorption temperatures. Thus, it seems evident that these two mass spectral signals correspond to two different products. These two signals in the mass 
spectrum are consistent with the empirical formulae $\mathrm{C}_{3} \mathrm{H}_{4} \mathrm{O}$ and $\mathrm{C}_{3} \mathrm{H}_{4} \mathrm{O}_{2}$ and so can clearly be identified with single and double addition of $\mathrm{O}$ atoms to propyne.

To determine the yield of each of these products, the total integrated mass spectral signals at $m / z=56$ and $m / z=72$ in each TPD measurement is determined. This procedure involves selecting the temperature range over which each product desorbs and summing the mass spectra in this temperature range for one TPD measurement to form an integrated mass spectrum. A background mass spectrum, recorded in an identical experiment carried out with the microwave discharge off, is then subtracted from this integrated mass spectrum. This background correction procedure confirms the product signals are a result of the oxygen atoms generated in the discharge. The intensities of the resulting mass spectral peaks are determined from this "corrected" integrated spectrum by peak fitting. This integration procedure is repeated for each separate experiment, and the results averaged for each surface temperature at which the dosing was carried out. The above integration procedure gives the relative ion signals at $\mathrm{m} / z=56$ and $m / z=72$ in terms of ion counts at the MCP detector. To compare with the kinetic model, with which we interpret our results (see below), we require the product yields in terms of the number of molecules formed on the surface. To transform our mass spectral ion counts to these absolute units requires calibration of the mass spectral intensities. To carry out this transformation, as reported before, ${ }^{\mathbf{8}, \mathbf{9}}$ we measure the mass spectrum resulting from a TPD dataset recorded following the adsorption of a known dose of propyne when the surface is held at $12 \mathrm{~K}$. The integrated propyne mass spectral intensity in such a TPD dataset allows us to calculate the proportionality constant, the detection efficiency, between the dose of molecules and the mass spectral signal, assuming a sticking probability of 1 in the calibration experiment. Such a sticking coefficient is likely to be an excellent characterization of the interaction of propyne with the $12 \mathrm{~K}$ surface. The detection efficiency we measure for propyne must now be adjusted to represent the detection of the two product molecules. Since the ionization cross sections at $200 \mathrm{eV}$ are not known for propyne and the two products $\left(\mathrm{C}_{3} \mathrm{H}_{4} \mathrm{O}\right.$ and $\left.\mathrm{C}_{3} \mathrm{H}_{4} \mathrm{O}_{2}\right)$, we must assume they are the same for all three species. Since we integrate just the parent ion signal in the mass spectrum, we must also adjust the detection efficiency to reflect the parent to fragment ion ratio in the mass spectrum of propyne and in the mass spectra of the product species. For the single addition product we used the parent to fragment ion ratio in a propanal mass spectrum measured in our apparatus. For the double addition product we estimated the fragment to parent ratio in the standard mass spectrum of methyl glyoxal from the NIST reference database. ${ }^{49}$ The above procedure allows us to estimate the yield of the product ions on an absolute scale from our TPD spectra, and report the yield as a function of the dosing temperature.

We initially report the total product yield, the summed yields of the single and double addition products, as a function of dosing surface temperature (Figure 2). These experimental results show that below $30 \mathrm{~K}$ the yield of $\mathrm{C}_{3} \mathrm{H}_{4} \mathrm{O}$ gently increases with decreasing temperature. Above a surface temperature of $30 \mathrm{~K}$ the product yield increases to a maximum at $50 \mathrm{~K}$ and then decreases to zero at a surface temperature of $100 \mathrm{~K}$. We also report the individual yields for the single addition product and the double addition product (Figure 3). Figure 3 shows that the same general trend with surface temperature is exhibited by the yields of both of the individual addition products as was described for the total product yield 
(Figure 2). We also note the yields for the single and double addition products are very similar below a surface temperature of $40 \mathrm{~K}$. Above a surface temperature of $40 \mathrm{~K}$ the yield of the single addition product is much larger than that of the double addition product (Figure 3).

\section{Data reduction}

To extract estimates of kinetic parameters from our experimental product yields, we fit the experimental data with a simple kinetic model. This kinetic model has been described before in the literature, ${ }^{\mathbf{8}, 9}$ but has also been extended here to model sequential addition of two oxygen atoms to propyne.

The kinetic model involves the two prototypical mechanisms by which the reaction of $\mathrm{O}$ atoms with propyne can proceed on the surface. Firstly, the LH mechanism where both reactants are adsorbed and thermalised on the substrate:

$$
\mathrm{C}_{3} \mathrm{H}_{4} \text { (ads) }+\mathrm{O}(\text { ads }) \rightarrow \mathrm{C}_{3} \mathrm{H}_{4} \mathrm{O}(\mathrm{ads})
$$

Secondly, the ER mechanism, where an adsorbed species undergoes reaction with a gas-phase partner:

$$
\mathrm{C}_{3} \mathrm{H}_{4}(\mathrm{ads})+\mathrm{O}(\mathrm{g}) \rightarrow \mathrm{C}_{3} \mathrm{H}_{4} \mathrm{O}(\mathrm{ads})
$$

In our experiments, as we have described before, ${ }^{8,9}$ the molecular species should have a significantly larger adsorption energy than the $\mathrm{O}$ atom. Thus, to simplify our model we only consider the form of ER reactivity where the $\mathrm{O}$ atom is the gas-phase reactant and the propyne molecule is adsorbed on the surface. This is because when both reactants are adsorbed on the surface, the rate for $\mathrm{LH}$ reactivity should dominate. Given the arguments above, the rates for the LH and ER mechanisms are therefore:

$$
\begin{gathered}
r_{\mathrm{LH}}=k_{\mathrm{LH}}\left[\mathrm{C}_{3} \mathrm{H}_{4}(\mathrm{ads})\right][\mathrm{O}(\mathrm{ads})] \\
r_{\mathrm{ER}}=k_{\mathrm{ER}}\left[\mathrm{C}_{3} \mathrm{H}_{4}(\mathrm{ads})\right] F_{\mathrm{O}}
\end{gathered}
$$

where $r_{i}$ is the rate of reaction and $k_{i}$ is the rate coefficient. In these equations $i$ denotes the $\mathrm{LH}$ or ER mechanism, $\left[\mathrm{C}_{3} \mathrm{H}_{4}(\mathrm{ads})\right]$ and $[\mathrm{O}(\mathrm{ads})]$ are the surface concentrations (in molecules $\mathrm{cm}^{-2}$ ) of the reactants absorbed on the surface and $F_{\mathrm{O}}$ is the deposition fluence of oxygen atoms.

At the low surface temperatures employed in this study, and given the inertness of the surface, it is safe to assume that the reactants are physisorbed and, as physisorption is dominant, there is no limit on the number of accessible adsorption sites. That is, once the first layer of molecular ice, comprising reactants and products, is deposited a second layer will then readily form on top of the first layer, eventually building up a multi-layer ice. In our model, we assume only the uppermost monolayer is accessible to the incident reactants, that is, we assume the reactants radicals cannot penetrate into the ice. Thus, the maximum surface coverage of each species is constrained to $10^{15}$ molecules $\mathrm{cm}^{-2}$ in our model, ${ }^{\mathbf{8} 9}$ the standard value for an accessible monolayer.

The temperature variation of the rate coefficients for the two surface reactions can be described by an Arrhenius expression: 


$$
k_{i}=A_{i} \exp \left(-E_{i} / R T\right)
$$

where $i$ again denotes the LH or ER mechanism. In equation $5, A_{i}$ is a pre-exponential factor and $E_{i}$ is the activation energy of the pathway. To simplify the modelling and reduce the number of free parameters, the activation energy is assumed to be the same for both the LH and ER mechanisms.

Since both LH and ER reactions can occur simultaneously, the overall rate $r$ of formation of the single addition product is given by:

$$
r=k_{\mathrm{LH}}\left[\mathrm{C}_{3} \mathrm{H}_{4}(\mathrm{ads})\right][\mathrm{O}(\mathrm{ads})]+k_{\mathrm{ER}}\left[\mathrm{C}_{3} \mathrm{H}_{4}(\mathrm{ads})\right] F_{\mathrm{o}}
$$

As we see in Eq. 6, the reaction rate is dependent upon the surface concentration of propyne and oxygen atoms. For propyne the three factors that control the surface concentration are given in Equation 7:

$$
\frac{d\left[\mathrm{C}_{3} \mathrm{H}_{4}\right]}{d t}=F_{\text {propyne }}-r-r_{\text {Des,propyne }}
$$

In equation $7, F_{\text {propyne }}$ is the flux of propyne onto the substrate and $r_{\text {Des,propyne }}$ is the rate of propyne desorption from the surface. The surface concentration of propyne can be found by integrating Equation 7 with respect to deposition time $t$. As described before, the propyne flux, $F_{\text {propyne, }}$ is estimated experimentally from the propyne flow rate and the pressure/pumping characteristics of the vacuum chamber. ${ }^{33,35}$ The depletion of propyne due to the reaction $r$ is calculated using equation 6 . Finally, the desorption rate of propyne, $r_{\text {Des,propyne, }}$ can be evaluated using a second Arrhenius equation at each surface temperature:

$$
r_{\text {Des,propyne }}=A_{\text {Des,propyne }} \exp \left(-E_{\text {Des,propyne }} / R T\right)\left[\mathrm{C}_{3} \mathrm{H}_{4}(\mathrm{ads})\right]
$$

where $A_{\text {Des,propyne }}$ is the pre-exponential factor for the desorption of propyne and $E_{\text {Des,propyne }}$ is the desorption energy for propyne. As discussed before, since the desorption of the multi-layer ice in our experiment just reveals another layer of the ice for reactions, only desorption in the monolayer regime will affect the surface concentrations and hence first-order desorption kinetics are used in Equation (9)..$^{33,35}$

The concentration of oxygen atoms is evaluated analogously to the concentration of propyne, allowing for the dissociation efficiency of the source. ${ }^{33,35}$ Desorption of the products of the reactions are not considered in our model. Such a simplification is justifiable as the products from the surface reaction between propyne and oxygen are heavier and more polar than the reactants and so should have larger desorption energies. This conclusion is confirmed by the TPD profiles we observe for the products.

As discussed above, the different TPD profiles of the two products, $\mathrm{m} / \mathrm{z}=56$ and $m / z=72$, confirm that the single addition signal, $m / z=56$, is from a separate product to that responsible for the double addition signal at $m / z=72$. That is, the signal at $m / z=56$ is clearly not a mass spectral fragment of the species at $\mathrm{m} / \mathrm{z}=$ 72. Given that the reaction conditions rule out a concerted three-body reaction, it seems clear that the reaction that forms the double addition product occurs in two steps. Firstly, a single oxygen atom adds to propyne to form $\mathrm{a}_{3} \mathrm{H}_{4} \mathrm{O}$ species. Then, in the second step, a second oxygen atom adds to the $\mathrm{C}_{3} \mathrm{H}_{4} \mathrm{O}$ to form the 
double addition product $\left(\mathrm{C}_{3} \mathrm{H}_{4} \mathrm{O}_{2}\right)$. Hence, all the double addition product we detect was at some point during the reaction a single addition product. Thus, to extract the kinetic parameters for the initial single addition step, we fit our kinetic model to the summed experimental yields of the single and double addition products.

Some preliminary considerations need to be addressed before fitting the kinetic model to the experimental data (Figure 2) in order to extract the kinetic parameters. The experimental yield curves we determined in previous investigations of the reactions of $\mathrm{O}$ displayed unambiguous evidence of the parallel operation of both the LH and ER mechanisms. ${ }^{9}$ Specifically, a clear peak in the yield was observed at surface temperatures just below the desorption temperature of the $\mathrm{O}$ atoms. At higher surface temperatures another peak in the yield was observed due to efficient operation of the ER pathway. The experimental yield profile for $\mathrm{O}+$ propyne (Figure 2) does not show this distinct double peaked structure. However it is apparent that the $\mathrm{LH}$ mechanism is still a significant contributor to the yield of $\mathrm{C}_{3} \mathrm{H}_{4} \mathrm{O}$, since we see a sharp drop in the yield at surface temperatures where the $\mathrm{O}$ atoms no longer stick efficiently to the surface, above approximately $50 \mathrm{~K}$. Our kinetic model does not give a good fit to the experimental data using the $\mathrm{LH}$ mechanism alone since above $50 \mathrm{~K}$, where the $\mathrm{O}$ atoms no longer stick efficiently, there is still a significant yield of $\mathrm{C}_{3} \mathrm{H}_{4} \mathrm{O}$. Therefore, although there is no observed double peaked structure in the experimental yield, both the LH and ER processes appear to be contributing to the product yield. Thus we have used both the LH and ER reactions in our kinetic model to fit the experimental data.

To model the yield of $\mathrm{C}_{3} \mathrm{H}_{4} \mathrm{O}$ at a given surface temperature, we numerically integrate Equation 6 for a time period equal to the experimental dosing period. The total number of product molecules formed can then be compared with the experimental data. To achieve a fit with the experimental data, we can vary the preexponential factors and the activation energy for the chemical reaction and also the desorption energies of the reactants. Again to constrain the number of free parameters, the pre-exponential factors for the desorption of the two reactants from the surface are kept fixed. Usually such pre-exponential factors $\left(A_{\text {Des,o }}\right.$ and $A_{\text {Des,propyne }}$ ) are taken as the vibrational frequency of the adsorbate-surface bond. In previous work we employed a value for $A_{\text {Des, }}$ of $3.10 \times 10^{12} \mathrm{~s}^{-1}$ and we employ this value again here. ${ }^{9,50}$ To the best of our knowledge there are no available surface vibrational frequencies for propyne, so for $A_{\text {Des,propyne }}$ we employ the value of $2.33 \times 10^{12} \mathrm{~s}^{-1}$, which is that calculated for an acetylene-graphite bond. ${ }^{9,51}$

We first attempted to fit the kinetic model to the experimental data, constraining the pre-exponential factors for the LH and ER mechanisms to be equal $\left(A_{\mathrm{LH}}=A_{\mathrm{ER}}\right)$ and also constraining the activation energies $\left(E_{\mathrm{LH}}=E_{\mathrm{ER}}\right)$ for both mechanisms to be equal. Under these constraints the fit to the experimental data is poor. From this poor fit of the highly constrained model we concluded that we needed a larger rate coefficient for the ER mechanism than for the LH mechanism. If the activation energy for surface diffusion of the $\mathrm{O}$ atoms is not significant, we would expect the activation energies for the LH and ER mechanisms to be equal. Thus, to allow a larger ER rate coefficient in the model we permit $A_{\mathrm{ER}}$ to take a different value to $A_{\mathrm{LH}}$. This additional degree of freedom allows us to achieve a much better, although not perfect, fit to the experimental data (Figure 2) as discussed below. In attempting this fitting it is clear that the barrier for the LH 
Table 1 Kinetic parameters characterizing the single addition of $\bigcirc$ atoms to propyne, to form the single addition product $\mathrm{C}_{3} \mathrm{H}_{4} \mathrm{O}$, as a function of surface temperature. As discussed in the text, these parameters have been extracted by fitting a kinetic model to the experimental data we record for this surface reaction

\begin{tabular}{lllll}
\hline $\begin{array}{l}\left(E_{i} / \mathrm{R}\right) / K i=\mathrm{LH} \\
\text { or ER }\end{array}$ & $\begin{array}{l}10^{16} A_{\mathrm{LH}} / \\
\mathrm{cm}^{2} \mathrm{molecule} \mathrm{s}^{-1} \mathrm{~s}^{-1}\end{array}$ & $\begin{array}{l}10^{16} A_{\mathrm{ER}} / \\
\mathrm{cm}^{2} \mathrm{molecule}^{-1} \mathrm{~s}^{-1}\end{array}$ & $\begin{array}{l}E_{\text {Des,propyne} /} \\
\mathrm{kJ} \mathrm{mol}^{-1}\end{array}$ & $\begin{array}{l}E_{\text {Des,o }} / \\
\mathrm{kJ} \mathrm{mol}^{-1}\end{array}$ \\
\hline $160 \pm 10$ & $0.95 \pm 0.2$ & $2.45 \pm 0.4$ & $20.8 \pm 0.3$ & $14 \pm 2$
\end{tabular}

mechanism is well constrained by the form of the data to $160 \pm 10 \mathrm{~K}$. Similarly, the fitting shows the propyne binding energy is also well constrained by the experimental data; we comment further on the $\mathrm{O}$ atom binding energy below. The desorption energies for propyne and oxygen are found to be $20.8 \pm 0.3 \mathrm{~kJ} \mathrm{~mol}^{-1}$ and $14.0 \pm 1.2 \mathrm{~kJ} \mathrm{~mol}^{-1}$ respectively.

The parameters for this satisfactory fit are reported in Table 1 and the fit is shown graphically in Figure 2. As is clear in Figure 2, the reported fit still does not satisfactorily reproduce experimental yield at $T=70 \mathrm{~K}$. Possible physical explanations for this poor fit at a surface temperature of $70 \mathrm{~K}$ include the presence of more than one oxygen atom binding site, as discussed further below.

As mentioned earlier, we have also extended our model to attempt to extract kinetic parameters for the double addition reaction. This extension involves the addition of two further rate equations for the formation of the double addition product from the single addition product:

$$
\begin{gathered}
r_{\mathrm{LH}}^{\prime}=k_{\mathrm{LH}}^{\prime}\left[\mathrm{C}_{3} \mathrm{H}_{4} \mathrm{O}(\mathrm{ads})\right][\mathrm{O}(\mathrm{ads})] \\
r_{\mathrm{ER}}^{\prime}=k_{\mathrm{ER}}^{\prime}\left[\mathrm{C}_{3} \mathrm{H}_{4} \mathrm{O}(\mathrm{ads})\right] F_{\mathrm{O}}
\end{gathered}
$$

Again, we integrate these equations to derive the yield of double addition product at the different surface temperatures, allowing the single addition product to form with the kinetic parameters listed in Table 1. Under these constraints we find that to fit the yield of the double addition product (Figure 3) requires a negative activation energy $(-60 \pm 20 \mathrm{~K})$.

\section{Discussion}

The oxygen atom desorption energy which gives the best fit to the experimental data in our kinetic model is $15.3 \mathrm{~kJ} \mathrm{~mol}^{-1}$. The interaction energy of an oxygen atom and pyrene (representative of a bridge site in graphite) is calculated to be $11.6 \mathrm{~kJ} \mathrm{~mol}^{-1} .^{50}$ As discussed above, in our experiments the graphite substrate is saturated after a matter of seconds during dosing. This means that although our substrate is graphite, the majority of the oxygen atoms are interacting with a propyne-oxygen matrix. Previous experimental work has shown that the desorption energy of an oxygen atom from an ethene-oxygen matrix and from a propene-oxygen matrix is, similarly to the oxygen-graphite system, about $12 \mathrm{~kJ}$ $\mathrm{mol}^{-1}$.9 The oxygen atom desorption energy which best fits our data is markedly larger than these previous values for the binding of oxygen atoms to small organic molecules. If we use a value of $12.9 \mathrm{~kJ} \mathrm{~mol}^{-1}$ in our simulation (Figure 4), we 


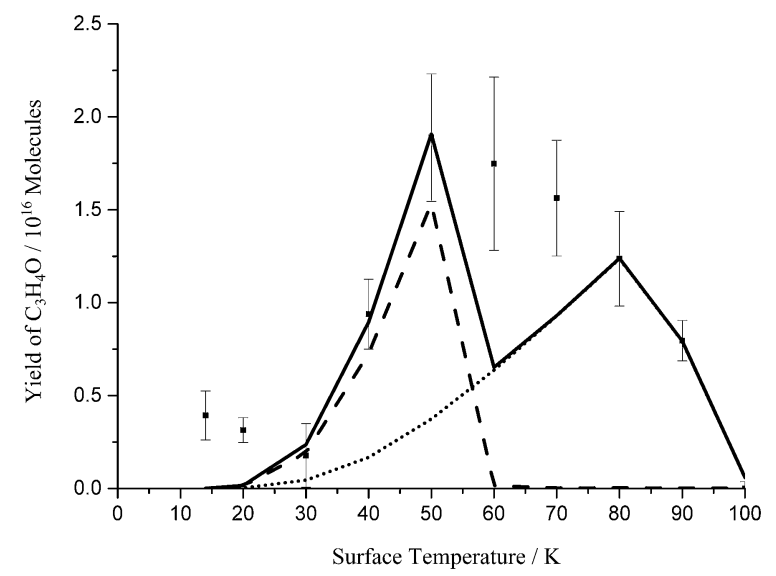

Fig. 4 Summed yield of the single and double addition product, formed following the codeposition of propyne $\left(\mathrm{C}_{3} \mathrm{H}_{4}\right)$ and $\mathrm{O}$ atoms, as a function of surface temperature. Squares: experimental data; solid line: model; dash LH mechanism; dot ER mechanism. The error bars associated with the experimental results represent two standard deviations from four repeats at each surface temperature. The kinetic model used to derive the fits shown employs the paramenters listed in Table 1 and an $\mathrm{O}$ atom desorption energy of $12.9 \mathrm{~kJ} \mathrm{~mol}^{-1}$.

satisfactorily reproduce the rise in the yield of the product over the surface temperature range from $30 \mathrm{~K}$ to $50 \mathrm{~K}$, but a larger desorption energy, or a component of the oxygen atoms possessing a larger desorption energy, is required to fit the data point at a surface temperature of $60 \mathrm{~K}$ (Figure 2). From this analysis it appears that the experimental data might be best represented with at least two desorption energies for the oxygen atoms. Given the consequent increase in the number of free parameters, we are reluctant to use such a desorption energy distribution for the oxygen atoms as we feel we risk over-interpreting our experimental data. We simply report that the oxygen atom desorption energy is not that well defined by the form of our experimental data, and is best represented by a value of $14 \pm 2 \mathrm{~kJ} \mathrm{~mol}^{-1}$, this value perhaps representing a distribution of desorption energies.

The propyne desorption energy extracted using our kinetic model is $20.8 \pm 0.3$ $\mathrm{kJ} \mathrm{mol}^{-1}$. To our knowledge there are no literature values for the desorption energy of propyne from interstellar ice analogues or a graphite surface. The value of this desorption energy agrees nicely with the observed trend that an alkyne's desorption energy is smaller than that of its alkene equivalent. ${ }^{51}$ Specifically, the work of Rubes et al. ${ }^{51}$ shows that ethene's desorption energy from a graphite (0001) surface is $17.36 \pm 0.0361 \mathrm{~kJ} \mathrm{~mol}^{-1}$ whilst acetylene's desorption energy from the same surface is $14.67 \pm 0.0273 \mathrm{~kJ} \mathrm{~mol}^{-1}$. In agreement with this trend, previous work has shown that the desorption energy of propene from propene ice is $21.4 \pm 0.3 \mathrm{~kJ} \mathrm{~mol}^{-1},{ }^{9}$ a value that is slightly larger than the equivalent value we extract here for the desorption of propyne.

Our modelling yields a value for the activation energy of single $\mathrm{O}$ atom addition to propyne of $160 \pm 10 \mathrm{~K}$, a value larger than the comparable reaction of $\mathrm{O}$ atoms with propene $(145 \pm 10 \mathrm{~K}) .{ }^{9}$ Such an increase in the activation energy is in accord with chemical intuition, as the reactive $\mathrm{C}-\mathrm{C}$ bond in propyne is stronger than that 
in propene. Such a trend is also supported by the fact we observe no reaction between $\mathrm{O}$ atoms and acetylene at a range of surface temperatures between $14 \mathrm{~K}$ and $100 \mathrm{~K}$. Previous work has shown that the activation energy (for the LH mechanism) for the reaction between ethene and $\mathrm{O}$ atoms is $190 \pm 45 \mathrm{~K} .{ }^{9}$ The absence of any signals in our experiments for the addition of $\mathrm{O}$ atoms to acetylene indicates the barrier for this process is significantly above $200 \mathrm{~K}$. Such a conclusion is in accord with the trend of a rise in the activation energy, for the reaction with $\mathrm{O}$ atoms, on changing the reactant from the alkene to the corresponding alkyne.

More generally, our results show that, under our surface conditions, the methyl substituted organic reactant (propene and propyne) is more reactive with oxygen atoms that the corresponding smaller molecule (ethene and acetylene). A similar trend is observed in combustion chemistry ${ }^{43}$ where the activation energy for the reaction of $\mathrm{O}$ atoms with propyne is smaller than that for acetylene.

The pre-exponential factors we extract from the fit of the kinetic model to the experimental data are $9.8 \pm 0.2 \times 10^{-17} \mathrm{~cm}^{1}$ molecule ${ }^{-1} \mathrm{~s}^{-1}$ and $2.45 \pm 0.4 \times 10^{-16}$ $\mathrm{cm}^{1}$ molecule $\mathrm{s}^{-1} \mathrm{~s}^{-1}$ for the $\mathrm{LH}$ and ER reactions respectively. These pre-exponential factors are approximately a factor of 10 smaller than we report for the reaction between oxygen atoms and alkenes. ${ }^{9}$ This trend in pre-exponential factors is also present in the gas phase, where the pre-exponential factors for the reaction of $\mathrm{O}$ atoms with alkynes are smaller than those for the reaction with alkenes. ${ }^{3,52}$ As encompassed by these pre-exponential factors, and discussed above, to fit our experimental data (Figure 2), the rate coefficient for the ER reaction must be larger than the rate coefficient for the LH process. One reason for this difference might be the fact that in the ER process the $\mathrm{O}$ atoms are not thermalized with the surface. Indeed, in our experiment the $\mathrm{O}$ atoms will possess a kinetic energy distribution representative of $300 \mathrm{~K}$. One way to test such a hypothesis experimentally would be to cool the incident $\mathrm{O}$ atom beam, but the methodology for such cooling is not well established experimentally. A greater rate constant for the ER mechanism, in comparison with the LH mechanism, has also been reported for the reactions between $\mathrm{O}$ atoms and $\mathrm{O}_{2}$ on cold surfaces and again assigned to non-thermal $\mathrm{O}$ atoms arriving at the surface. ${ }^{36}$

As discussed above, at surface temperatures below $30 \mathrm{~K}$ the yield of the single and double addition products increases as the surface temperature decreases.
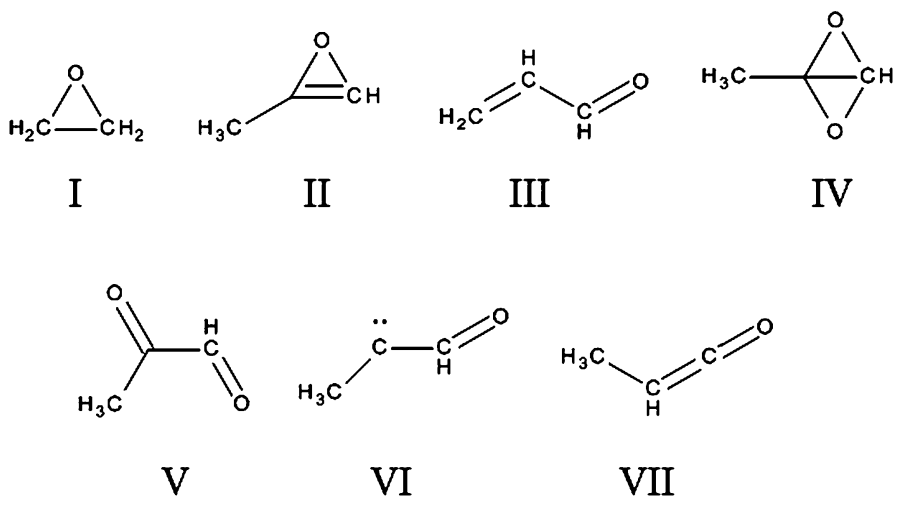

Fig. 5 Organic structures relevant to the discussion of the reaction of $O$ atoms with propyne. 
Our simple kinetic model does not account for the increase in the product yield at temperatures below $30 \mathrm{~K}$. A similar reactive pathway, with an increasing yield at low temperatures, has been observed before for reactions of $\mathrm{O}$ atoms with $\mathrm{CS}_{2}{ }^{8}$ Reactivity at such surface temperatures is particularly pertinent to the ISM. Since the rate of this low temperature channel rises with falling surface temperature, it could be that this low temperature process is effectively barrierless. Since this low temperature reactivity is only present between surface temperatures of $14 \mathrm{~K}$ and $30 \mathrm{~K}$, performing any sort of kinetic fit for our data is not statistically meaningful. Hence we simply report that the rate coefficient at $14 \mathrm{~K}$ for the formation of the single addition product is $(6 \pm 2) \times 10^{-19} \mathrm{~cm}^{1}$ molecule ${ }^{-1} \mathrm{~s}^{-1}$. We note that this low temperature rate is at least comparable to that observed for the reaction of $\mathrm{O}$ atoms with $\mathrm{CS}_{2},{ }^{8}$ and that tunneling processes have been recently reported for $\mathrm{O}$ atoms at low surface temperatures. ${ }^{36}$

Our key finding for the addition of a second oxygen atom to the $\mathrm{C}_{3} \mathrm{H}_{4} \mathrm{O}$ single addition product is that this is a "barrierless" process. Such a conclusion is not unexpected since the initial addition of an oxygen atom to propyne is likely to initially result in a radical species. The subsequent reaction of this radical with a further oxygen atom might be expected to exhibit a negative activation energy. ${ }^{53}$

As shown in Figure 3, the yields of the single $\left(\mathrm{C}_{3} \mathrm{H}_{4} \mathrm{O}\right)$ and double $\left(\mathrm{C}_{3} \mathrm{H}_{4} \mathrm{O}_{2}\right)$ addition products are similar at surface temperatures below $40 \mathrm{~K}$. Above $40 \mathrm{~K}$ there is a dramatic increase in the yield of the single addition product with respect to the double addition product. The observed increase in the yield of the single addition product at surface temperatures above $40 \mathrm{~K}$ perhaps implies that the reactive intermediate resulting from single addition isomerizes above $40 \mathrm{~K}$ to yield a more stable isomer of $\mathrm{C}_{3} \mathrm{H}_{4} \mathrm{O}$, an isomer that is less susceptible to attack by a second oxygen atom.

Our previous work has shown that the surface reaction between ethene $\left(\mathrm{C}_{2} \mathrm{H}_{4}\right)$ and $\mathrm{O}$ atoms results in the formation of ethylene oxide (Structure I, Figure 5). ${ }^{9}$ Similarly it is reasonable to propose that the first intermediate formed by the reaction of $\mathrm{O}$ atoms with propyne is an oxirene (II, Figure 5). We would expect this oxirene to be reactive, but potentially to be stabilized on a low temperature surface. Such an interpretation agrees nicely with our deduction above that at surface temperatures below $40 \mathrm{~K}$ we have a reactive single addition product on the surface, but above a surface temperature of $40 \mathrm{~K}$ this reactive single addition product isomerizes to a less reactive species. There are many (10) possible isomerisation products for the reactive oxirene, but several of these would also be highly reactive. However, one stable molecule which can be formed from the isomerisation of the oxirene is propenal (III, Figure 5), the isomerisation occurring via a pathway involving a carbene..$^{54}$ It has been shown that the rearrangement of an oxirene to a ketocarbene is barrierless. ${ }^{55}$ Indeed, the first investigation of the gas-phase reactivity of $\mathrm{O}$ atoms with propyne concluded that, if energy could be efficiently lost from the initial addition product, the ketocarbene (VI Figure 5) formed from the oxirene will relax to methyl ketene (VII Figure 5) or to propenal (III Figure 5). ${ }^{38}$

The structure of the double addition product is more speculative. An obvious candidate for the initial product of the second addition is the bicyclic structure IV (Figure 5). This strained intermediate might be expected to be very reactive and will probably rapidly rearrange. There are a number of possible rearranged structures for this primary bicyclic adduct, with one stable rearrangement product being methyl glyoxal (V Figure 5). 


\section{Astrophysical implications}

Our experiments show that on interstellar dust grain analogues, under UHV conditions and at low temperature, oxygen atoms can readily add to the carboncarbon triple bond of a propyne molecule. The reaction is detectable at surface temperatures of $14 \mathrm{~K}$ and above, and is most efficient at $50 \mathrm{~K}$. We estimate our experiments to be the equivalent of approximately $10^{5}-10^{6}$ years of exposure to $\mathrm{O}$ atoms in an interstellar cloud. ${ }^{9,33,35}$ It is therefore possible that this pathway is active in interstellar clouds. In addition, given the observed kinetics, the yield from the reaction of propyne with oxygen atoms may also be significant when the cloud begins to warm. ${ }^{56}$

Our experiments reveal a marked difference in the heterogeneous reactivity of acetylene and propyne on low temperature surfaces, a difference in reactivity that may be pertinent in the ISM. Indeed, we note that the reactions of molecular ices with incident oxygen atoms are not well represented in recent descriptions of gasgrain reaction networks. ${ }^{57}$

As discussed in the Introduction, propyne has been observed in a variety of environments in the ISM, and in these environments the surface chemistry we report in this article may be relevant. We also previously discussed the need for an $\mathrm{O}$ atom sink in interstellar dust clouds, to account for the observed gas-phase depletion. We postulate that the stable molecule resulting from the single addition of $\mathrm{O}$ atoms to propyne on a cold surface is propenal (III Figure 5). This reaction, along with other $\mathrm{O}$ atom reactions, may contribute to that $\mathrm{O}$ atom sink. Propenal $\left(\mathrm{C}_{3} \mathrm{H}_{4} \mathrm{O}\right)$ has been observed in the ISM towards the star forming region Sagittarius B2(N). ${ }^{58}$ Hollis et al. postulate that the formation of interstellar propenal is due to hydrogen addition to propynal..$^{58}$ However, the work reported in this paper suggests that, in addition, the surface chemistry of propyne may also be a source of propenal. Clearly modelling work is required to support this suggestion. Encouragingly, propyne has also been detected in Sagittarius B2 ${ }^{59}$ in accord with the idea that propenal may be formed from the heterogeneous oxidation of propyne by oxygen atoms.

To the best of our knowledge no molecule with the empirical formula of the double addition product $\left(\mathrm{C}_{3} \mathrm{H}_{4} \mathrm{O}_{2}\right)$ has been observed in the interstellar medium. However, given the observed facility of the double addition process, our experimental data would suggest that at least one isomer of $\mathrm{C}_{3} \mathrm{H}_{4} \mathrm{O}_{2}$, perhaps methylglyoxal, could be present during the warm-up phase of an interstellar cloud where propyne ice has been thermally processed by oxygen atoms.

\section{Conclusions}

This paper reports the first laboratory investigation of the heterogeneous reaction of propyne with oxygen atoms at temperatures relevant to the ISM. Our data shows this reaction, which initially forms an adduct due to single addition $\left(\mathrm{C}_{3} \mathrm{H}_{4} \mathrm{O}\right)$ proceeds efficiently at surface temperatures below $100 \mathrm{~K}$. As the surface temperature decreases from $100 \mathrm{~K}$ the yield of the reaction increases to reach a maximum at $50 \mathrm{~K}$ and then falls to a minimum at $30 \mathrm{~K}$. Modelling the yield of the reaction at surface temperatures above $30 \mathrm{~K}$ allows the extraction of an activation energy for the reaction of $160 \pm 10 \mathrm{~K}$. We also detect a product corresponding to the addition of two oxygen atoms to propyne. This double-addition product 
appears to form in a sequential reaction of the single addition product with another oxygen atom. The addition of this second oxygen atom to the single addition product appears to be barrierless. We propose structures for the intermediates and products of the $\mathrm{O}+$ propyne reaction which are consistent with our observations and the known behaviour of these organic compounds. We postulate that the addition of oxygen atoms to propyne to form propenal could act as a source of this molecule in the ISM.

\section{Acknowledgements}

We would like to thank University College London and the Max-Planck Institute for Astronomy for support of HJK. We also acknowledge support from the European Community's Seventh Framework Programme (FP7/2007-2013) under the LASSIE training network. We also gratefully acknowledge very helpful discussions with Eric Herbst, Serena Viti, Wendy Brown, Michael Ward and Angela Occhiogrosso.

\section{References}

1 S. Kwok and Y. Zhang, Nature, 2011, 479, 80-83.

2 Y. J. Kuan, S. B. Charnley, H. C. Huang, W. L. Tseng and Z. Kisiel, Astrophys. J., 2003, 593, 848-867.

3 A. G. G. M. Tielens, The Physics and Chemistry of the Interstellar Medium, Cambridge Univ. Press, Cambridge, 1st ed edn., 2005.

4 D. J. Burke and W. A. Brown, Phys. Chem. Chem. Phys., 2010, 12, 5947-5969.

5 L. R. Nittler and C. M. O. D. Alexander, Highlights of Astronomy, 2006, 14, 17-20.

6 D. A. Williams and E. Herbst, Surf. Sci., 2002, 500, 823-837.

7 H. J. Fraser, M. R. S. McCoustra and D. A. Williams, Astron. Geophys., 2002, 43, 10-18.

8 M. D. Ward, I. A. Hogg and S. D. Price, Mon. Not. R. Astron. Soc., 2012, 425, 1264-1269.

9 M. D. Ward and S. D. Price, Astrophysical Journal, 2011, 741.

10 B. E. Turner, E. Herbst and R. Terzieva, Astrophys. J. Suppl., 2000, 126, 427-460.

11 S. Martin, R. Mauersberger, J. Martin-Pintado, C. Henkel and S. Garcia-Burillo, Astrophys. J. Suppl., 2006, 164, 450-476.

12 E. Churchwell and J. M. Hollis, Astrophys. J., 1983, 272, 591-608.

13 E. Herbst and E. F. van Dishoeck, in Annual Review of Astronomy and Astrophysics, Vol 47, eds. R. Blandford, J.Kormendy and E. VanDishoeck, Annual Reviews, Palo Alto, 2009, vol. 47, pp. 427-480.

14 A. Fuente, S. Garcia-Burillo, M. Gerin, D. Teyssier, A. Usero, J. R. Rizzo and P. de Vicente, Astrophys. J., 2005, 619, L155-L158.

15 R. Mauersberger, C. Henkel, C. M. Walmsley, L. J. Sage and T. Wiklind, Astronomy \& Astrophysics, 1991, 247, 307-314.

16 S. Maity and R. I. Kaiser, Astrophysical Journal, 2013, 773.

17 K. Hiraoka, N. Mochizuki and A. Wada, in Astrochemistry: From Laboratory Studies to Astronomical Observations, eds. R. I. Kaiser, P. Bernath, Y. Osamura, S. Petrie and A. M. Mebel, 2006, vol. 855, pp. 86-99.

18 T. Kasamatsu, T. Kaneko, T. Saito and K. Kobayashi, Bull. Chem. Soc. Jpn., 1997, 70, 1021-1026. 
19 Y. Dede and I. Ozkan, Phys. Chem. Chem. Phys., 2012, 14, 2326-2332.

20 Y. Takano, H. Masuda, T. Kaneko and K. Kobayashi, Chem. Lett., 2002, 986987, DOI: $10.1246 / \mathrm{cl} .2002 .986$.

21 T. Fouchet, E. Lellouch, B. Bezard, H. Feuchtgruber, P. Drossart and T. Encrenaz, Astronomy and Astrophysics, 2000, 355, L13-L17.

22 T. deGraauw, H. Feuchtgruber, B. Bezard, P. Drossart, T. Encrenaz, D. A. Beintema, M. Griffin, A. Heras, M. Kessler, K. Leech, E. Lellouch, P. Morris, P. R. Roelfsema, M. RoosSerote, A. Salama, B. Vandenbussche, E. A. Valentijn, G. R. Davis and D. A. Naylor, Astronomy and Astrophysics, 1997, 321, L13-L16.

23 Y. Alibert, O. Mousis and W. Benz, Astrophys. J., 2005, 622, L145-L148.

24 Y. Alibert, O. Mousis, C. Mordasini and W. Benz, Astrophys. J., 2005, 626, L57L60.

25 D. Gautier, F. Hersant, O. Mousis and J. I. Lunine, Astrophys. J., 2001, 559, L183-L183.

26 D. Gautier, F. Hersant, O. Mousis and J. I. Lunine, Astrophys. J., 2001, 550, L227-L230.

27 F. Hersant, D. Gautier and J. I. Lunine, Planet. Space Sci., 2004, 52, 623-641.

28 O. Mousis, Y. Alibert and W. Benz, Astron. Astrophys., 2006, 449, 411-415.

29 M. Burgdorf, G. Orton, J. van Cleve, V. Meadows and J. Houck, Icarus, 2006, 184, 634-637.

30 A. Coustenis, B. Bezard, D. Gautier, A. Marten and R. Samuelson, Icarus, 1991, 89, 152-167.

31 N. A. Teanby, P. G. J. Irwin, R. de Kok, A. Jolly, B. Bezard, C. A. Nixon and S. B. Calcutt, Icarus, 2009, 202, 620-631.

32 S. I. B. Cartledge, J. T. Lauroesch, D. M. Meyer and U. J. Sofia, Astrophys. J., 2004, 613, 1037-1048.

33 U. Hincelin, V. Wakelam, F. Hersant, S. Guilloteau, J. C. Loison, P. Honvault and J. Troe, Astronomy \& Astrophysics, 2011, 530.

34 D. C. B. Whittet, Astrophys. J., 2010, 710, 1009-1016.

35 E. B. Jenkins, Astrophys. J., 2009, 700, 1299-1348.

36 M. Minissale, E. Congiu, S. Baouche, H. Chaabouni, A. Moudens, F. Dulieu, M. Accolla, S. Cazaux, G. Manico and V. Pirronello, Physical Review Letters, 2013, 111.

37 S. L. Zhao, W. Q. Wu, H. M. Zhao, H. Wang, C. F. Yang, K. H. Liu and H. M. Su, J. Phys. Chem. A, 2009, 113, 23-34.

38 C. A. Arrington and D. J. Cox, J. Phys. Chem., 1975, 79, 2584-2586.

39 G. Y. Adusei, A. S. Blue and A. Fontijn, J. Phys. Chem., 1996, 100, 16921-16924.

40 E. N. Aleksandrov, I. V. Dubrovina and S. N. Kozlov, Kinet. Catal., 1981, 22, 394-396.

41 J. M. Brown and B. A. Thrush, Trans. Faraday Soc., 1967, 63, 630.

42 K. H. Homann and C. Wellmann, Ber. Bunsen-Ges. Phys. Chem., 1983, 87, 527532.

43 J. R. Kanofsky, D. Lucas, F. Pruss and D. Gutman, J. Phys. Chem., 1974, 78, 311316.

44 G. Q. Xing, X. Huang, X. B. Wang and R. Bersohn, J. Chem. Phys., 1996, 105, 488-495.

45 M. E. Umstead, R. G. Shortridge and M. C. Lin, Chem. Phys., 1977, 20, 271-276.

46 H. E. Avery and S. J. Heath, J. Chem. Soc., Faraday Trans. 1, 1972, 68, 512. 
47 H. p. Schreibe and A. g. Mackinno, Can. J. Chem., 1968, 46, 1033.

48 M. Hawkins and L. Andrews, J. Am. Chem. Soc., 1983, 105, 2523-2530.

49 N. M. S. D. Center and D. S. E. Stein, in NIST Chemistry WebBook, NIST Standard Reference Database Number 69, Eds. P.J. Linstrom and W.G. Mallard, National Institute of Standards and Technology, Gaithersburg MD, 20899, http:// webbook.nist.gov, (retrieved December 4, 2013).

50 H. Bergeron, N. Rougeau, V. Sidis, M. Sizun, D. Teillet-Billy and F. Aguillon, J. Phys. Chem. A, 2008, 112, 11921-11930.

51 M. Rubes, J. Kysilka, P. Nachtigall and O. Bludsky, Phys. Chem. Chem. Phys., 2010, 12, 6438-6444.

52 R. Atkinson and R. j. Cvetanov, J. Chem. Phys., 1972, 56, 432-437.

53 I. R. Sims and I. W. M. Smith, Annu. Rev. Phys. Chem., 1995, 46, 109-137.

54 T. S. S. Rao and S. Awasthi, THEOCHEM, 2008, 848, 98-113.

55 J. P. Toscano, M. S. Platz and V. Nikolaev, J. Am. Chem. Soc., 1995, 117, 47124713.

56 A. Occhiogrosso, S. Viti, M. D. Ward and S. D. Price, Mon. Not. R. Astron. Soc., 2012, 427, 2450-2456.

57 P. Theule, F. Duvernay, G. Danger, F. Borget, J. B. Bossa, V. Vinogradoff, F. Mispelaer and T. Chiavassa, Adv. Space Res., 2013, 52, 1567-1579.

58 J. M. Hollis, P. R. Jewell, F. J. Lovas, A. Remijan and H. Mollendal, Astrophys. J., 2004, 610, L21-L24.

59 S. L. E. D. Buhl, Molecules in the Galactic Environment, Wiley-Interscience, New York, 1973. 